\title{
A Deformation Tracking Approach to 4D Coronary Artery Tree Reconstruction
}

\author{
Yanghai Tsin ${ }^{1}$, Klaus J. Kirchberg ${ }^{1}$, Guenter Lauritsch ${ }^{2}$, and Chenyang $\mathrm{Xu}^{1}$ \\ 1 Siemens Corporate Research, Princeton, NJ, USA \\ 2 Siemens Healthcare, Forchheim, Germany \\ first_name.last_name@siemens.com
}

\begin{abstract}
This paper addresses reconstruction of a temporally deforming 3D coronary vessel tree, i.e., 4D reconstruction from a sequence of angiographic X-ray images acquired by a rotating C-arm. Our algorithm starts from a 3D coronary tree that was reconstructed from images of one cardiac phase. Driven by gradient vector flow (GVF) fields, the method then estimates deformation such that projections of deformed models align with X-ray images of corresponding cardiac phases. To allow robust tracking of the coronary tree, the deformation estimation is regularized by smoothness and cyclic deformation constraints. Extensive qualitative and quantitative tests on clinical data sets suggest that our algorithm reconstructs accurate $4 \mathrm{D}$ coronary trees and regularized estimation significantly improves robustness. Our experiments also suggest that a hierarchy of deformation models with increasing complexities are desirable when input data are noisy or when the quality of the 3D model is low.
\end{abstract}

\section{Introduction}

A four dimensional (4D) representation of the coronary artery tree - i.e., a temporally deforming 3D coronary artery model, would have many interesting applications, e.g., motion compensated tomographic reconstruction, stenosis detection [1], foreshortening-free visualization, accurate measurement of cardiac motion parameters, and motion-based heart disease diagnosis. Automatic 4D reconstruction is challenging using traditional $2 \mathrm{D}$ cardiac monoplane or biplane angiograms due to difficulties with calibration and limited baselines, as shown by several prior studies [234]. This paper introduces a 4D reconstruction method using intra-operative rotational C-arm systems. Compared to biplane X-ray, rotational X-ray on a C-arm is clinically more prevalent, less costly, and it enables tomographic reconstruction.

$4 \mathrm{D}$ reconstruction seems to have a straightforward solution: by repeating 3D reconstruction for every cardiac phase. However, this is not a viable solution in practice for several reasons. First, independent 3D reconstruction results in the loss of vessel branch correspondences, which are necessary for a diagnostically useful time-dynamic model. The second reason is related to the rotational acquisition protocol. As opposed to biplane X-ray, no two images are acquired at 
exactly the same heart phase. A small temporal offset can cause a significant spatial error for fast motion cardiac phases (e.g. in early systole [5]), rendering $3 \mathrm{D}$ reconstruction impractical. This is less of an issue for slowly moving cardiac phases. Indeed, there is research aimed at finding the optimal cardiac phase that are best for $3 \mathrm{D}$ coronary tree reconstruction [6].

In this work, starting from a successfully reconstructed 3D model [7] for a relatively static cardiac phase, we explicitly estimate coronary tree deformations from one cardiac phase to the next. Since we track deformations of the 3D coronary tree, this is a $4 D$ reconstruction by deformation tracking approach.

State-of-the-art work in coronary artery tree reconstruction was done by Blondel et al. [1. The authors use 4D B-solids to model coronary motion with reported computation times of 15 to 30 minutes. Our approach is distinct in the following important ways. First, we derive the external force from gradient vector flow $(G V F)$ [8] computed from the vessel enhancement filter responses ( vesselness") 9]. GVF was proven to have superior capture range and convergence behavior over a regular gradient. It is also known that GVF is robust to noise perturbations, while $2 \mathrm{D}$ centerline extraction is extremely sensitive to noise, rendering centerlinebased methods unstable in noisy sequences. Second, we adopted more robust motion models (rigid and affine), involving $90 \sim 180$ parameters, compared to the B-solid motion model used in [1] involving 30,000 parameters. Complex models have two known disadvantages, 1) costly to optimize; and more importantly 2) prone to over-fit noise. We show in our experiments that simpler $4 \mathrm{D}$ reconstruction algorithm is able to handle structural perturbations (due to missing branches) and heavy image noise perturbations more stably.

A biplane 4D reconstruction method is reported in 10 . Besides differences in the image acquisition procedures (thus different problems), the external driving forces (GVF versus gradient-based potential field) and data representations are different. In our work, we work directly on 3D point sets while the method in [10 requires a B-spline fitting preprocessing step that brings additional challenges such as selection of control points for B-spline and detecting bifurcation points. In the approach presented by Jandt et al. [11, the 4D motion vector field of the coronary tree is recovered by correspondence matching of multi-phase 3D centerline models. In contrast, our method circumvents the aforementioned inherent inaccuracy of 3D models reconstructed for high-velocity cardiac phases (see above) by requiring only one baseline 3D reconstruction. Furthermore, our approach solves the challenging correspondence problem implicitly.

Motion estimation is a well-studied topic in the computer vision community, e.g. 12 and 13 . Our problem differs from the traditional tracking problem in that image plane motions are induced by two major sources: the rotation of the projection plane around the patient, and nonrigid cardiac deformation. In our problem, the former is known given the calibrated C-arm rotation. What is left to be estimated is the cardiac deformation. In addition, a X-ray image is in its projective nature different from a photometric image, as it can be seen as a superposition of multiple transparent layers. 2D motion field estimation thus requires different techniques. 


\section{Method}

In the following we denote vectors and matrices as boldface letters, e.g., $\mathbf{R}$. A letter with a tilde means that it is in a homogeneous coordinate, $\widetilde{\mathbf{X}}_{n}^{(p)}=$ $\left[\mathbf{X}_{n}^{(p) T}, 1\right]^{T}$. A letter with a prime, e.g., $\mathbf{u}^{\prime}, \mathbf{X}^{\prime}$ represents deformation updated version of the same point (in $2 \mathrm{D}$ and $3 \mathrm{D}$ ), $\mathbf{u}, \mathbf{X}$.

Problem Formulation. We are given a sequence of $F$ X-ray images, $\mathcal{I}=\left\{\mathbf{I}_{f}\right\}$ and their corresponding projection matrices $\left\{\mathbf{P}_{f}\right\}, f=1,2, \ldots, F$, taken by an X-ray C-arm. Recorded ECG signals allow us to map each frame $f$ into one of $P$ discrete cardiac phases, $p=0,1, \ldots, P-1$. A 3D model of the coronary artery tree is reconstructed from a heart phase involving a low amount of motion. Without loss of generality, we term the optimal phase the base phase and denote it as phase 0 . The model is in the form of a set of $N 3 \mathrm{D}$ points, $\mathcal{X}^{(0)}=\left\{\mathbf{X}_{n}^{(0)}\right\}$, $n=1,2, \ldots, N$. The problem is to infer a temporally deforming coronary artery tree, $\mathcal{X}^{(p)}$ for all cardiac phases, from the images, such that projections of $\mathcal{X}^{(p)}$ align with observations in all image frames corresponding to the cardiac phase.

Deformation Models. In this study, we model deformation of a coronary tree by two 3D parametric deformation models, i.e., rigid and affine. The affine model can capture the majority of the beating motion associated with the cardiac cycle. Both transformations can be represented by a compact $4 \times 4$ matrix $\mathbf{T}(\boldsymbol{\theta})$, where $\boldsymbol{\theta}$ is the vector of motion parameters. Forward and inverse mappings between $\mathbf{T}$ and $\boldsymbol{\theta}$ are assumed understood [14, Ch. 1.4 pp. 16].

A 4D Coronary Tree Model. Then deformation from phase $p-1$ to $p$ is

$$
\widetilde{\mathbf{X}}_{n}^{(p)}=\mathbf{T}\left(\boldsymbol{\theta}^{(p-1)}\right) \cdot \widetilde{\mathbf{X}}_{n}^{(p-1)}
$$

Note that all points in a $3 \mathrm{D}$ model $\mathbf{X}^{(p-1)}$ are deformed to the next phase by the same $\mathbf{T}$. By applying (1) recursively, we have

$$
\widetilde{\mathbf{X}}_{n}^{(p)}=\left(\prod_{i=p-1}^{0} \mathbf{T}\left(\boldsymbol{\theta}^{(i)}\right)\right) \cdot \widetilde{\mathbf{X}}_{n}^{(0)} \doteq \mathcal{T}^{(p)}(\boldsymbol{\theta}) \cdot \widetilde{\mathbf{X}}_{n}^{(0)}
$$

where $\mathcal{T}^{(p)}(\boldsymbol{\theta}) \doteq \prod_{i=p-1}^{0} \mathbf{T}\left(\boldsymbol{\theta}^{(i)}\right)$ is the accumulative deformation from frame 0 to $p$ and $\boldsymbol{\theta}=\left(\boldsymbol{\theta}^{(0) T}, \ldots, \boldsymbol{\theta}^{(P-1) T}\right)^{T} \in \mathcal{R}^{m P \times 1}$ is a concatenation of $\boldsymbol{\theta}^{(p)}$, and $m$ is the parameter length (6 for rigid and 12 for affine).

Deformation Update. We adopt an iterative deformation update approach. Initially, the $4 \mathrm{D}$ coronary tree is trivial, i.e., $\mathcal{X}^{(p)}=\mathcal{X}^{(0)}, \forall p$. Due to cardiac deformations, projections of this trivial 4D coronary reconstruction will not align with the observed X-ray images except for the base phase. The iterative update approach then seeks to find updates to $\mathbf{T}^{(p)}, \forall p$, such that the projections progressively match towards the image observations. In the following, we will use a compositional update rule: 


$$
\mathbf{T}\left(\boldsymbol{\theta}^{(p)}\right) \leftarrow \mathbf{T}\left(\delta \boldsymbol{\theta}^{(p)}\right) \cdot \mathbf{T}\left(\boldsymbol{\theta}^{(p)}\right) .
$$

Put all the update parameters in a vector $\delta \boldsymbol{\theta} \doteq\left(\delta \boldsymbol{\theta}^{(0) T}, \ldots, \delta \boldsymbol{\theta}^{(P-1) T}\right)^{T} \in$ $\mathcal{R}^{m P \times 1}$ and by slight abuse of notation, denote

$$
\mathcal{T}^{(p)}(\boldsymbol{\theta}, \delta \boldsymbol{\theta}) \doteq \prod_{i=p-1}^{0}\left(\mathbf{T}\left(\delta \boldsymbol{\theta}^{(i)}\right) \cdot \mathbf{T}\left(\boldsymbol{\theta}^{(i)}\right)\right)
$$

as the updated accumulative deformation. An updated 2D projection $\widetilde{\mathbf{u}}^{\prime}$ (in homogeneous coordinate) is derived by

$$
\widetilde{\mathbf{u}}_{n f}^{\prime}(\boldsymbol{\theta}, \delta \boldsymbol{\theta}) \doteq\left[x_{n f}^{\prime}, y_{n f}^{\prime}, z_{n f}^{\prime}\right]^{T} \cong \mathbf{P}_{f}^{(p)} \cdot \mathcal{T}^{(p)}(\boldsymbol{\theta}, \delta \boldsymbol{\theta}) \cdot \widetilde{\mathbf{X}}_{n}^{(0)} .
$$

where $\cong$ means equal up to a scale. Note that the phase-frame correspondence $p \leftrightarrow f$ is known given the recorded ECG signal. Next, the updated 2D projection in nonhomogeneous coordinate is

$$
\mathbf{u}_{n f}^{\prime}=\left[\frac{x_{n f}^{\prime}}{z_{n f}^{\prime}}, \frac{y_{n f}^{\prime}}{z_{n f}^{\prime}}\right]^{T} \approx \mathbf{u}_{n f}+\left[\begin{array}{l}
\mathbf{d}_{1 n f} \\
\mathbf{d}_{2 n f}
\end{array}\right] \cdot \delta \boldsymbol{\theta}
$$

where in the second (approximate) equality we used first order approximations and $\mathbf{d}_{k n f}, k=1,2$ are data term related Jacobian for point $n$ in frame $f$. Denote

$$
\mathbf{v}_{n f} \doteq\left[v_{1 n f}, v_{2 n f}\right]^{T} \doteq \mathbf{u}_{n f}^{\prime}-\mathbf{u}_{n f}
$$

as the desired vector flow in a $2 \mathrm{D}$ image. Thus for each point $n$ and a frame $f$, we have derived two linear constraints on the deformation update parameters,

$$
\mathbf{d}_{k n f}^{T} \cdot \delta \boldsymbol{\theta} \approx v_{k n f}, \quad k=1,2 .
$$

In this study, we choose the gradient vector flow (GVF) field to provide $v_{k n f}$ due to many known advantages as stated previously in this paper.

Cyclic Deformation Constraints. Cardiac motions are cyclic, which implies that after a complete cycle, a point should end up at the same starting point,

$$
\mathcal{T}^{(P)}(\boldsymbol{\theta}, \delta \boldsymbol{\theta}) \widetilde{\mathbf{X}}_{n}^{(0)}=\widetilde{\mathbf{X}}_{n}^{(0)} .
$$

A first order approximation can be derive for each point $\mathbf{X}_{n}^{(0)}$

$$
\mathbf{c}_{k n}^{T} \cdot \delta \boldsymbol{\theta} \approx c_{k n}, \quad k=1,2,3
$$

each for a coordinate $X, Y, Z$. The right hand side has an intuitive explanation of cyclic residues, i.e., cyclic motion residue due to currently estimated deformation model $\boldsymbol{\theta}$. The above constraints express the requirement to make up for these residues using the deformation update $\delta \boldsymbol{\theta}$.

Smooth Deformation Constraints. Cardiac motions are smooth, which can be modeled by a Laplacian constraint,

$$
\left(\mathcal{T}^{(p-1)}(\boldsymbol{\theta}, \delta \boldsymbol{\theta})-2 \cdot \mathcal{T}^{(p)}(\boldsymbol{\theta}, \delta \boldsymbol{\theta})+\mathcal{T}^{(p+1)}(\boldsymbol{\theta}, \delta \boldsymbol{\theta})\right) \cdot \widetilde{\mathbf{X}}_{n}^{(0)}=0 .
$$


Again, a first order approximation can be derived,

$$
\mathbf{s}_{k n}^{(p) T} \cdot \delta \boldsymbol{\theta} \approx s_{k n}^{(p)}, \quad k=1,2,3 .
$$

The above constraints also has an intuitive interpretation: The deformation update (left) should make up for any non-smooth deformation (right side) due to the currently estimated deformation $\boldsymbol{\theta}$.

The Cost Function. Finally, by combining (8), (10), and (12), a cost function for $4 \mathrm{D}$ coronary tree reconstruction can be written as

$$
\mathcal{C}(\delta \boldsymbol{\theta})=\sum_{f, n, k}\left(\left\|\mathbf{d}_{k n f}^{T} \delta \boldsymbol{\theta}-v_{k n f}\right\|+\lambda_{s}\left\|\mathbf{s}_{k n}^{(p) T} \delta \boldsymbol{\theta}-s_{k n}^{(p)}\right\|+\lambda_{c}\left\|\mathbf{c}_{k n}^{T} \delta \boldsymbol{\theta}-c_{k n}\right\|\right)
$$

where $\lambda_{s}$ and $\lambda_{c}$ are weights for smoothness and cyclic constraints respectively. We use $L_{2}$ norm in this study. (13) can be solved efficiently using least-squares.

\section{Results}

The X-ray sequences for our experiments were acquired with a Siemens AXIOMArtis C-arm system featuring a digital flat panel detector. The system has a constant source intensifier distance (SID), constant cranio/caudal angle and varying anterior/oblique angle, covering a range of 220 degrees. It is run in rotational acquisition mode, which is commonly used to reconstruct CT-like volumes. The $\mathrm{C}$-arm is calibrated so that the perspective projection matrix for each image is known accurately and the acquisition is done under patient breath-hold. A contrast dye is injected into the coronary arteries immediately preceding the acquisition. In addition, the ECG signal is recorded in sync with the image sequence, which allows retrospective gating.

We designed the experiments to test the limits of our $4 \mathrm{D}$ reconstruction algorithm along two dimensions. Along the first dimension, we tested the influence of structural perturbation, utilizing 3D models of different quality and detail. The experiments were conducted with four 3D models on three distinct sequences of X-ray images. The first 3D model was constructed using a set of manually labeled vessel centerlines, which resulted in the best quality input 3D model (but still not perfect). The other 3D models were reconstructed fully automatically. The last 3D model contained only two branches that were almost coplanar, an ill-conditioned case for complex deformation models, including the affine model. Ground-truth vessel centerline segmentation was provided for quantitative evaluation of the first two experiments. All GVF fields were automatically computed from vesselness images 9. We tested the algorithms on four variations: un-regularized $\left(\lambda_{s}=\lambda_{c}=0\right)$ rigid and affine, regularized rigid and affine. For all regularized methods, we used fixed regularization parameters $\lambda_{s}=0.1$ and $\lambda_{c}=1.0$. A cardiac cycle was divided into 15 discrete cardiac phases, i.e, $P=15$.

Along the second dimension, we studied the influence of noise perturbations. We added zero mean Gaussian noise over 10 noise-levels $(\sigma=0,25, \ldots, 225)$ to the X-ray images, whereas the input images have a dynamic range of 16 bit and 


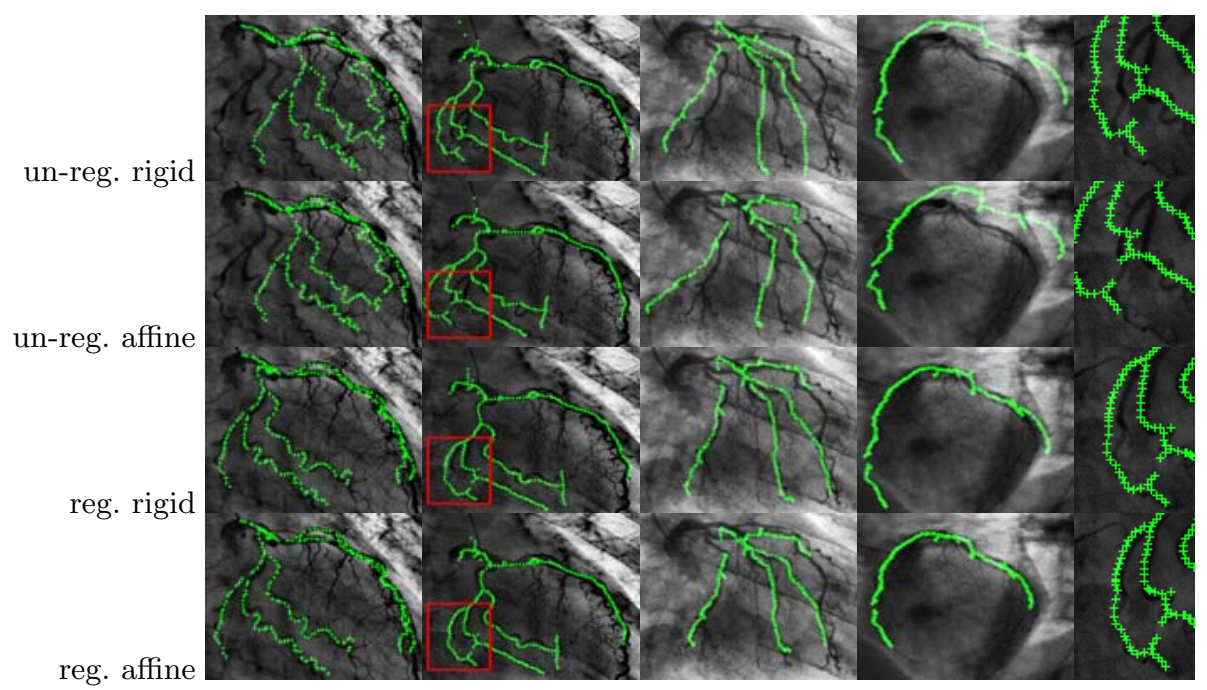

Fig. 1. 4D reconstruction results on the four 3D models. The regularized versions (the last two rows) outperformed the un-regularized versions (the first two rows). The last column shows enlarged views of red box bounded regions of the second column.

the gray value contrast of the major vessels ranged between 50 and 600 . For each noise level, we conducted 20 trials of reconstruction using all four methods. For the first two experiments, we conducted 724 reconstructions each.

A qualitative overview of reconstruction results is provided in Figure 1, In general the regularized versions worked better than the non-regularized versions. For the first three cases, we could always find frames that regularized versions succeeded while the non-regularized versions failed, but not the other way around. For the last (ill-conditioned) case, however, we observed that 4D reconstruction using regularized affine was not stable. We could observe rapid shrinkage and expansion of a branch along the true vessel. This was a signature of an over-complex deformation model and noise overfitting.

For the first two experiments, where ground-truth vessel centerlines were available, we measured the $2 \mathrm{D}$ projection errors: the distances from projections of points on the $4 \mathrm{D}$ model to the nearest centerline point. Overall, 2D projection error has standard deviations about $3.2 \mathrm{~mm}$ for the un-regularized versions and $1.9 \mathrm{~mm}$ for the regularized versions. For the first case, the majority $(72 \%)$ of the points have a projection error of less than $3 \mathrm{~mm}$. Figure 2 shows errors averaged over all points, all frames and all 20 trials. The black line at the bottom shows the baseline error: average $2 \mathrm{D}$ projection error of the base phase. We can draw similar conclusions for both experiments. 1) The regularized versions resulted in lower 2D projection errors. 2) With good quality input, a more complex deformation model (affine) resulted in better reconstruction accuracy. 3) Over a large range of noise levels, the reconstruction accuracy did not suffer for the regularized versions. In addition to the effect of the regularization terms, the superior 

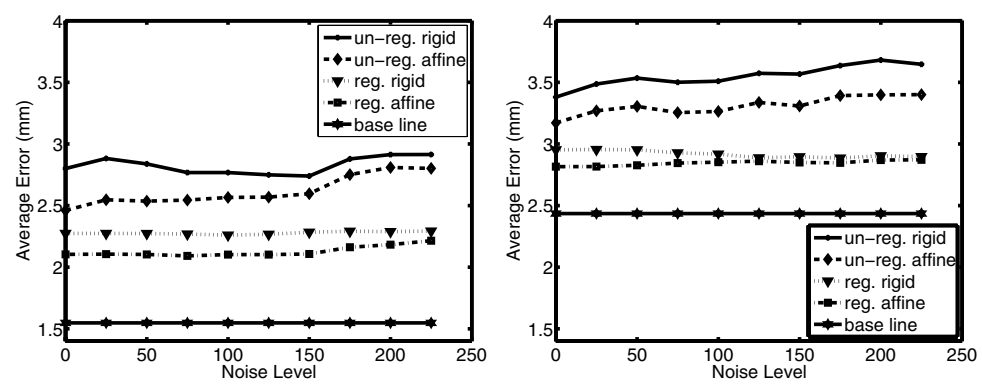

Fig. 2. Average 2D error. Left, using the best 3D model constructed from manually labeled vessel centerlines. Right, starting from a fully automatic $3 \mathrm{D}$ reconstruction.

noise-resistance capability of GVF helped. Our experiments show that even a moderate noise perturbation can change centerline extraction dramatically, rendering centerline-based methods [1] unstable.

To further understand how robust the algorithms were with respect to noise perturbation, we studied the variance $\hat{\sigma}^{2}$ of a reconstructed point $\mathbf{X}_{n, t}^{(p)}$ over all trials $t$. The variance is then averaged over all points and all three dimensions to give an average measurement. The first observation was that in all cases the simpler rigid model was more stable than the affine model (standard deviation $\hat{\sigma}$ on average $37 \%$ smaller). Also, as expected, the regularized versions were much more stable than the un-regularized counterparts ( $\hat{\sigma}$ on average $66 \%$ smaller). Second and more interestingly, in the ill-conditioned case, regularized affine model resulted in a comparable standard deviation to un-regularized rigid, indicating instability of the former. It can be assumed that models more complex than affine would have suffered even more.

Finally, note that all 3D models contained less than 300 points, providing less than 600 constraints per frame. The B-solid model of Blondel et al. [1] required 1000 variables per frame, far larger than the number of constraints. Very strong regularization terms would be required for their method to succeed on our data. In the first experiment, deformation updates (13) converged in less than 10 seconds for the un-regularized methods, 30 and 90 seconds for regularized rigid and affine respectively, compared to $15 \sim 30$ minutes reported in [1].

\section{Conclusions}

We proposed a 4D coronary tree reconstruction by deformation tracking method in this paper. Its effectiveness is shown by our extensive experiments. Key elements of our method are 1) GVF as external force; 2) the regularization terms. Fast computation time makes this approach usable in an intra-operative scenario.

We also find that for well-conditioned problems, complex models matching true deformations provide better reconstruction accuracy. However, for ill-posed problems, simpler models or a hierarchical approach are preferred. The right 
balance between model complexity, data degeneracy and noise-level can be formally addressed by statistical model selection theories, e.g., Geometric-AIC [15], which we leave as a future research topic.

\section{References}

1. Blondel, C., Malandain, G., Vaillant, R., Ayache, N.: Reconstruction of coronary arteries from a single rotational X-ray projection sequence. IEEE TMI 25(5), 653-663 (2006)

2. Chen, S., Carroll, J.: 3-D reconstruction of coronary arterial tree to optimize angiographic visualization. IEEE TMI 19(4), 318-336 (2000)

3. Chen, S.Y., Carroll, J.: Kinematic and deformation analysis of 4-D coronary arterial trees reconstructed from cine angiograms. IEEE TMI 22(6), 710-721 (2003)

4. Andriotis, A., Zifan, A., Gavaises, M., et al.: A new method of three-dimensional coronary artery reconstruction from X-ray angiography: validation against a virtual phantom and multislice computed tomography. Catheter Cardio Inte. 71(1), 28-43 (2008)

5. Husmann, L., Leschka, S., Desbiolles, L., et al.: Coronary artery motion and cardiac phases: dependency on heart rate - implications for CT image reconstruction. Radiology 245(2), 567-576 (2007)

6. Hansis, E., Schaefer, D., Doessel, O., Grass, M.: Automatic optimum phase point selection based on centerline consistency for 3D rotational coronary angiography. IJCARS 3(3), 355-361 (2008)

7. Liao, R., Sun, Y., Duong, L.: 3-D symbolic reconstruction of coronary artery tree from multiple views of rotational X-ray angiography. In: Proc. MICCAI-CVII, pp. 80-87 (2008)

8. Xu, C., Prince, J.: Snakes, shapes, and gradient vector flow. IEEE TIP 7(3), 359369 (1998)

9. Frangi, A.F., Niessen, W.J., Vincken, K.L., Viergever, M.A.: Multiscale vessel enhancement filtering. In: Wells, W.M., Colchester, A.C.F., Delp, S.L. (eds.) MICCAI 1998. LNCS, vol. 1496, pp. 130-137. Springer, Heidelberg (1998)

10. Shechter, G., Devernay, F., Coste-Maniere, E., et al.: Three-dimensional motion tracking of coronary arteries in biplane cineangiograms. IEEE TMI 22(4), 493-503 (2003)

11. Jandt, U., Schäfer, D., Grass, M., Rasche, V.: Automatic generation of time resolved motion vector fields of coronary arteries and 4D surface extraction using rotational x-ray angiography. Phys. Med. Biol. 54(1), 45-64 (2009)

12. Bergen, J.R., Anandan, P., Hanna, K.J., Hingorani, R.: Hierarchical model-based motion estimation. In: Sandini, G. (ed.) ECCV 1992. LNCS, vol. 588, pp. 237-252. Springer, Heidelberg (1992)

13. Hager, G.D., Belhumeur, P.N.: Efficient region tracking with parametric models of geometry and illumination. IEEE TPAMI 20(10), 1025-1039 (1998)

14. Hartley, R.I., Zisserman, A.: Multiple View Geometry in Computer Vision, 1st edn. Cambridge University Press, Cambridge (2000)

15. Kanatani, K.: Statistical Optimization for Geometric Computation: Theory and Practice, 1st edn. North-Holland, Amsterdam (1996) 